\title{
INVENTORY MODEL FOR DETERIORATING ITEMS WITH QUADRATIC DEMAND, PARTIAL BACKLOGGING AND PARTIAL TRADE CREDIT
}

\author{
D. Sharmila ${ }^{1}$ and R.Uthayakumar ${ }^{2}$ \\ Department of Mathematics, The Gandhigram Rural Institute - Deemed University, \\ Gandhigram - 624 302. Dindigul, Tamil Nadu, India.
}

\section{SUMMARY}

This article develops inventory model for deteriorating items with quadratic demand. Shortages are allowed. We study the partial delay in payments. The objective is to find the optimal cycle times that minimize the total cost. In this study first, we developed arithmetical model and procedure of finding the most favorable solution is developed. Also the solution process is developed in order to minimize the total cost. The total costs are calculated by various principles. A solution process is developed to find the optimal solution and statistical examples are presented to demonstrate the result of the proposal model. Compassion study of the most favorable solution with respect to the parameters of the system is carried out and recommendations for further research are provided.

\section{KEYWORDS:}

Quadratic demand, deterioration, shortages, partial trade credit. Subject Classification Code: $90 B 05$

*Corresponding author Tel. +91-451-2452371, Fax: 91-451-2453071

\section{PROLOGUE}

Inventory management is the administration of supply, storage and convenience of items in order to ensure a sufficient supply without excessive oversupply. It can also be referred as domestic control - an accounting method or system designed to promote efficiency or assure the implementation of a policy or safeguard assets or avoid fraud and error etc. The main target of inventory organization deals with minimization of the inventory carrying costs for which it is necessary to determine the optimal stock and optimal time of refill for the future. Researchers had developed inventory models by assuming quadratic demand for items like fruits, vegetables, materials, iron goods, clothes, etc., since the demand rate is flexible due to buyers and sellers.

Due to the customer's choice and the arrival of new products may decline the demand rate. Attempting the obvious fact of time-varying demand pattern in the deteriorating inventory models yields very much real time application. Inventory has two types based on time - varying demands namely discrete and continuous time. The continuous - time inventory models were developed the demand pattern by linearly increasing or decreasing.

DOI : $10.5121 /$ oraj.2015.2404 
Operations Research and Applications : An International Journal (ORAJ), Vol.2, No.4, November 2015

throughout their life time owed to delay, injure, spoilage, consequence of the other reasons. Yet to be paid to this fact, calculating and maintaining the inventory of deteriorating items becomes a tough problem for the assessments.

If the suppliers offered the customers the permissible delay in payments if the order quantity is less than the predetermined quantity and such system is termed as partial trade credit. The partial trade credit is one of the central features in supply chain management. In real life situation this partial trade credit is more matched to the retailer.

The willingness of the customer to wait for shortages with the length of the waiting time. Here shortages are as backlogging; the impact on the cost from the delay of the products can be balanced out.

\section{LITERATURE REVIEW}

Abubakar Musa et.al [1] described several deteriorating items that do not start deteriorating immediately they were held in stock. And also reduction of these items as soon as they are stored would depend on demand, and when deterioration begins, depend on both demand and deterioration. Aggarwal [2] discussed an inventory model for a system with charge is time dependent. Amutha and Chandrasekaran [3] developed model with constant deterioration rate and time dependent holding cost. R. Begum et al. [5] developed the model with time dependent on quadratic demand rate.

K.Annadurai and R.Uthayakumar [4] developed the partial trade credit financing in a supply chain by EOQ - based model for decomposing items together with shortages and also they discussed about partial permissible delay in payments even if the order quantity is less than the pre-determined quantity.

Chaintanya Kumar Tripathy et at.[6] another model with quadratic demand when deterioration rate depends on two parameters and also discussed to be the subject to time depend Weibull deterioration, they include the shortages and replenishment is infinite. Deb et at. [7] studied an inventory for assuming the shortages and found how the profit was. Ghare [8] studied about the demand which is decaying exponential. Goyal and Giri [9] methodically review the recent trend in deteriorating inventory models. Inventory model for the most favorable pricing and ordering policies for the vendor with trade credit was formulated by Hwang and Shinn [11] they have suggested the condition that the supplier offers trade credit to the purchaser if the order quantity is greater than or equal to a pre determined quantity.

Mitra et al. [12] an inventory model for constant demand and the deteriorating items and also they included the variable holding cost considering the shortage costs. Mohan andVenkateswarlu [13] developed an EOQ model for deteriorating products by considering rate of demanded is quadratic with respect to time. Narayan Singh et al. developed a three echelon supply chain inventory model with permissible delay in payments. Soni $\mathrm{H}$ et al. [17] analyzed by agree to the shortages and the partial trade credit financing in a supply chain by EOQ-based model for decaying items. Liao [14] developed an EOQ model with non instantaneous acknowledgment and exponential deteriorating item under two-level trade credit. 
Operations Research and Applications : An International Journal (ORAJ), Vol.2, No.4, November 2015

Hardik N. Soni [10] recognized an inventory model for deteriorating items under stock- needy demand and two-level trade credit and obtained the optimal replenishment policy. Sarkar et.al [15] described with a most favorable inventory refill policy for a deteriorating item considering time-quadratic demand and time-dependent partial backlogging which depends on the length of the waiting time for the next replenishment over a finite time horizon and variable replenishment cycle. There is no inventory model, by EOQ - based model for deteriorating items with partial trade credit under quadratic demand with shortages. This paper is organized as follows. We described the required EOQ - based inventory model with partial trade credit and also formulated several derivations. In this paper we consider the demand as quadratic form. By this form we gauge the total costs. To highlights and visualize the results, examples are given. Then sensitivity psychotherapy is carried out and some executive insights are obtained.

Vandana and Sharma [18] developed an economic production quantity inventory model for noninstantaneous deteriorating item sunder trade credit policy. Vandana and Sharma [19] prepared an inventory model for non- instantaneous deteriorating items with quadratic demand rate and shortages under trade credit policy.

In this article, we suppose that the inventory model for deteriorating items with quadratic demand partial backlogging and partial trade credit. The inventory is assumed to deteriorate at a stable rate and shortage is allowed. In this study, first we developed a mathematical model and procedure for finding the optimal solutions and our approach is illustrated through a numerical example. We provide the entries and hypothesis for the proposed model in section 3. Arithmetic model is established in section 4. A section 5 describes the solution process. Statistical examples are provided to illustrate the proposed model in section 6.The compassion study of the most favorable solution with respect to parameters of the system is carried out in Section 7. Finally, we present the termination and future research in section 8 .

\section{ENTRIES AND HYPOTHESIS}

The mathematical model in this paper is developed on the basis of the following entries and hypothesis

\subsection{Entries}

To expand the proposed model, we accept the following entries

A

$\mathrm{D}$

Q

$\mathrm{T}$

$t_{1}$

$b_{1}$

$a_{1}$

$w$

d
Setup costs or ordering costs per order

Annual demand (i.e., $a+b t+c t^{2} ; a \neq 0 ; b \neq 0 ; c \geq 0$ )

order quantity

Inventory cycle length

Length of time (with no shortages)

Backlogging parameter

The deterioration rate

The measure at which the full delay payment acceptable per order the little bit of the delay payments acceptable by the provider per order 
Operations Research and Applications : An International Journal (ORAJ), Vol.2, No.4, November 2015

$\begin{array}{ll}C_{0} & \text { Unit purchase cost of the item } \\ C_{1} & \text { Shortage cost for backlogged item } \\ C_{2} & \text { Unit cost of lost sales } \\ \mathrm{M} & \text { delay period offered by the seller for settling the accounts to the vendor } \\ I_{e} & \text { Annual interest which can be earned } \\ I_{p} & \text { Annual interest payable } \\ \mathrm{P} & \text { Defective rate in an order lot } \\ I_{\max } & I_{1}(0)=I_{\text {max }} \\ T C_{1}\left(t_{1}, T_{1}\right) & \text { if } M \leq t_{1} \\ T C_{2}\left(t_{1}, T_{2}\right) & \text { if } t_{w} \leq t_{1} \leq M \\ T C_{3}\left(t_{1}, T_{3}\right) & \text { if } 0<t_{1}<t_{w}\end{array}$

\subsection{Hypothesis}

The following hypotheses are used in the development of the model:

1. The rate of demand is assumed to be in quadratic form.

2. Shortages are allowed and backlogged

3. The longer waiting time is ,the smaller the backlogging rate will be .Let $B(t)=\frac{1}{1+b_{1}(T-t)}$ where $t$ is the waiting time up to the next refill with $0 \leq b_{1} \leq 1$

4. The sellers may offer the partial delay in payments even if the order quantity is less than the pre-determined quantity.

5. The total annual cost $T C\left(t_{1}, T\right)=$ Ordering cost + Holding cost + Opportunity cost + Shortages cost due to backlogging + Deterioration cost + Cost of interest charges for the unsold items after the permissible delay in payments - Cost of interest earned from the last sales during the permissible period.

\section{ARITHMETICAL MODEL}

An inventory model, a arithmetical model to find the most favorable refill in the cycle time and to minimize the total annual cost is developed. 
Operations Research and Applications : An International Journal (ORAJ), Vol.2, No.4, November 2015

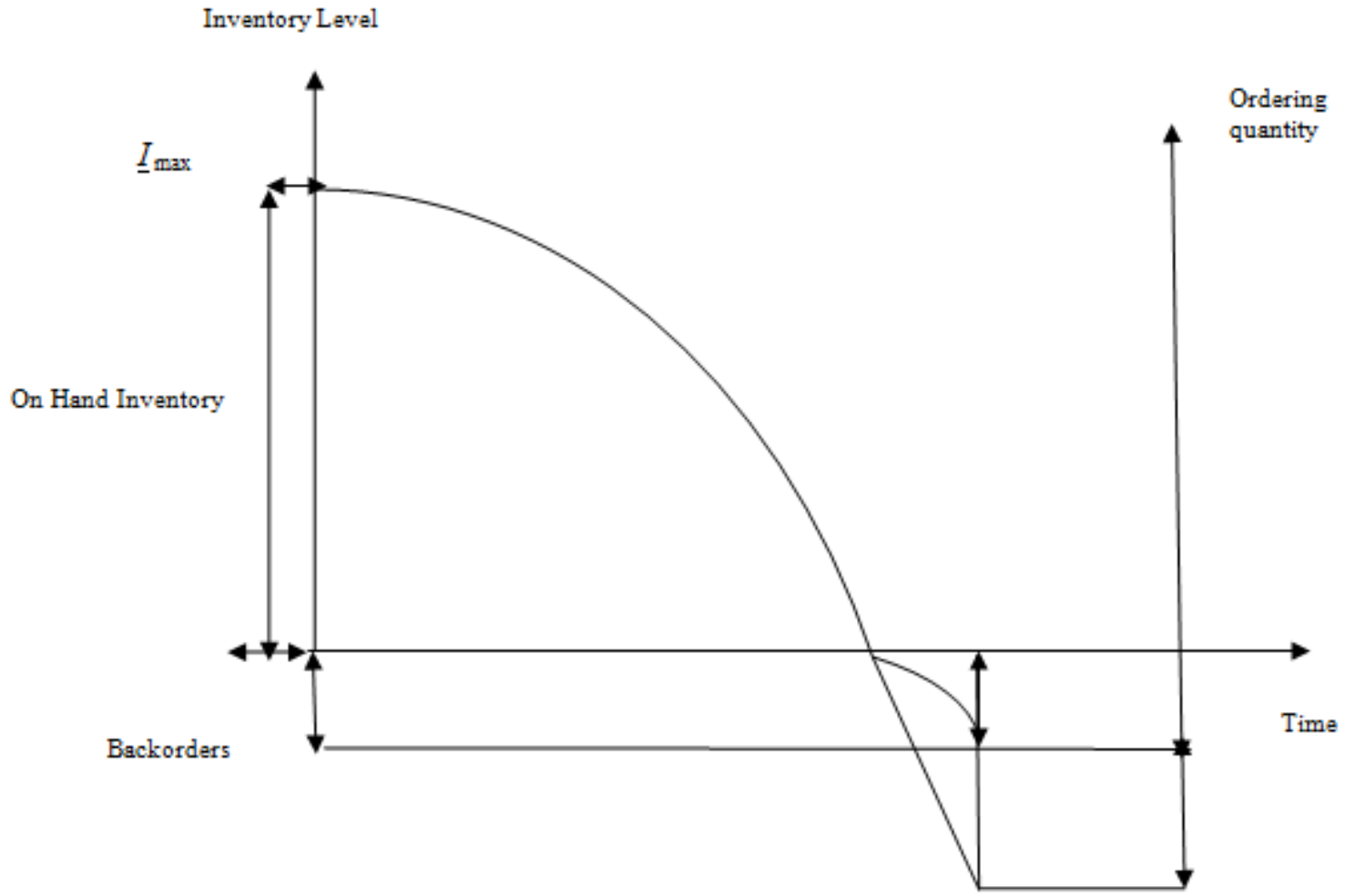

Figure 1 Graphical image of inventory system with shortages

Let $I_{1}(t)$ be the inventory at time $t_{1}\left(0 \leq t \leq t_{1}\right)$. Hence the differential equation representing the inventory status is given by,

$\frac{d I_{1}(t)}{d t}+a_{1} I_{1}(t)=-\left(a+b t+c t^{2}\right)$

With frontier condition $I_{1}(0)=I_{\max }$

The solution of equation (1) is given by

$$
I_{1}(t)=-\frac{b t}{a_{1}}-\frac{c t^{2}}{a_{1}}+\frac{2 c t}{a_{1}^{2}}-\frac{a}{a_{1}}+\frac{b}{a_{1}^{2}}-\frac{2 c}{a_{1}^{3}} \quad \text { if } 0 \leq t \leq t_{1}
$$

So, the maximum inventory altitude for each cycle can be obtained as 
Operations Research and Applications : An International Journal (ORAJ), Vol.2, No.4, November 2015

$$
\begin{aligned}
I_{\max }=I_{1}(0) & =\left(\frac{1}{a_{1}}\left[\frac{b}{a_{1}}-a-\frac{2 c}{a_{1}^{2}}\right]\right) \\
I_{1}(t) & =\left(\frac{2 c t}{a_{1}^{2}}+\frac{b}{a_{1}^{2}}-\frac{b t}{a_{1}}-\frac{c t^{2}}{a_{1}}-\frac{a}{a_{1}}-\frac{2 c}{a_{1}^{3}}\right)
\end{aligned}
$$

Shortage interval the demand at $t$ is partially backlogged at the fraction $B(t)=\frac{1}{1+b_{1}(T-t)}$.

Thus the differential equation leading the amount of demand backlogged is as below.

$$
\frac{d I_{2}(t)}{d t}=\frac{-\left(a+b t+c t^{2}\right)}{1+b_{1}(T-t)} ; t_{1} \leq t \leq T
$$

With frontier condition $I_{2}\left(t_{1}\right)=0$

The solution of equation (4) is given by

$$
\begin{aligned}
I_{2}(t) & =a\left(t_{1}-t\right)+a b_{1} T\left(t_{1}-t\right)+\frac{a b_{1}}{2}\left(t_{1}^{2}-t^{2}\right) \\
& +\frac{b}{2}\left(t_{1}^{2}-t^{2}\right)-\frac{b b_{1} T}{2}\left(t_{1}^{2}-t^{2}\right)+\frac{b b_{1}}{2}\left(t_{1}^{3}-t^{3}\right)+\frac{c}{3}\left(t_{1}^{3}-t^{3}\right)-\frac{c b_{1} T}{3}\left(t_{1}^{3}-t^{3}\right)
\end{aligned}
$$

if $t_{1} \leq t \leq T$

Thus we have,

$$
I(t)= \begin{cases}-\left(\frac{b t}{a_{1}}+\frac{c t^{2}}{a_{1}}-\frac{2 c t}{a_{1}^{2}}+\frac{a}{a_{1}}-\frac{b}{a_{1}^{2}}+\frac{2 c}{a_{1}^{3}}\right) & \text { if } 0 \leq t \leq t_{1} \\ a\left(t_{1}-t\right)+a b_{1} T\left(t_{1}-t\right)+\frac{a b_{1}}{2}\left(t_{1}^{2}-t^{2}\right) & \\ +\frac{b}{2}\left(t_{1}^{2}-t^{2}\right)-\frac{b b_{1} T}{2}\left(t_{1}^{2}-t^{2}\right)+\frac{b b_{1}}{2}\left(t_{1}^{3}-t^{3}\right) & \text { if } t_{1} \leq t \leq T \\ +\frac{c}{3}\left(t_{1}^{3}-t^{3}\right)-\frac{c b_{1} T}{3}\left(t_{1}^{3}-t^{3}\right) & \text { i }\end{cases}
$$

By letting $\mathrm{t}=\mathrm{T}$ in equation (5), we can obtain the maximum amount of demand backlogged per cycle as 
Operations Research and Applications : An International Journal (ORAJ), Vol.2, No.4, November 2015

$$
\Lambda=-I_{2}(T)=-\left(\begin{array}{l}
a\left(t_{1}-T\right)+a b_{1} T\left(t_{1}-T\right)+\frac{a b_{1}}{2}\left(t_{1}^{2}-T^{2}\right) \\
+\frac{b}{2}\left(t_{1}^{2}-T^{2}\right)-\frac{b b_{1} T}{2}\left(t_{1}^{2}-T^{2}\right)+\frac{b b_{1}}{2}\left(t_{1}^{3}-T^{3}\right) \\
+\frac{c}{3}\left(t_{1}^{3}-T^{3}\right)-\frac{c b_{1} T}{3}\left(t_{1}^{3}-T^{3}\right)
\end{array}\right)
$$

Hence the order quantity per cycle is given by

$$
Q=I_{\max }+\Lambda=\left(\frac{1}{a_{1}}\left[\frac{b}{a_{1}}-a-\frac{2 c}{a_{1}^{2}}\right]\right)-\left(\begin{array}{l}
a\left(t_{1}-T\right)+a b_{1} T\left(t_{1}-T\right)+\frac{a b_{1}}{2}\left(t_{1}^{2}-T^{2}\right) \\
+\frac{b}{2}\left(t_{1}^{2}-T^{2}\right)-\frac{b b_{1} T}{2}\left(t_{1}^{2}-T^{2}\right)+\frac{b b_{1}}{2}\left(t_{1}^{3}-T^{3}\right) \\
+\frac{c}{3}\left(t_{1}^{3}-T^{3}\right)-\frac{c b_{1} T}{3}\left(t_{1}^{3}-T^{3}\right)
\end{array}\right)
$$

We can obtain the time interval that w units are exhausted to zero due to the both demand and the deterioration as $t_{w}=\frac{1}{a_{1}} \log \left(\frac{a_{1} w}{a+b t+c t^{2}}+1\right)$.

If the fully delayed payment is permitted then the inequality $Q \geq w$ iff $t_{1} \geq t_{w}$.If $Q<w$ then the vendor must take a loan with the interest charged $I_{p}$.To pay the supplier the partial payment $(1-d) C_{0} Q$ when the order is filled at time zero. From the constant pays revenue the retailer will be able to pay off the loan $(1-d) C_{0} Q$ at time $\frac{(1-d) C_{0}}{P}\left(\frac{e^{a_{1} t_{1}}-1}{a_{1}}\right)$.

Now, the total annual cost of the inventory model for the vendor is computed using the following various components.

1. Ordering cost $=\frac{A}{T}$

2. Holding cost $=-\left[\frac{h}{T a_{1}}\left(a t_{1}+\frac{b t_{1}^{2}}{2}+\frac{c t_{1}^{3}}{3}-\frac{b t_{1}}{a_{1}}-\frac{c t_{1}^{2}}{a_{1}}+\frac{2 c t_{1}}{a_{1}^{2}}\right)\right]$

3. Deterioration cost $=-\left[\frac{C_{0}}{T}\left(a t_{1}+\frac{b t_{1}^{2}}{2}+\frac{c t_{1}^{3}}{3}-\frac{b t_{1}}{a_{1}}-\frac{c t_{1}^{2}}{a_{1}}+\frac{2 c t_{1}}{a_{1}^{2}}\right)\right]$ 
Operations Research and Applications : An International Journal (ORAJ), Vol.2, No.4, November 2015

4. Shortage cost due to backlogging $=-\frac{C_{1}}{T}\left[a\left(\left(\frac{-a T^{2}}{2}\right)+\frac{a b_{1} T^{3}}{2}-\frac{a b_{1} T^{3}}{6}-\frac{b T^{3}}{6}+\frac{b b_{1} T^{4}}{6}\right)\right.$

$$
\left.-\left(\frac{-a t_{1}^{2}}{2}+\frac{a b_{1} T t_{1}^{2}}{2}-\frac{a b_{1} t_{1}^{3}}{6}-\frac{b t_{1}^{3}}{6}+\frac{b t_{1}^{3} b_{1}}{6}\right)\right]
$$

5. Opportunity cost due to lost sales $=\frac{C_{2}}{T}\left[a\left(\frac{b_{1} T^{2}}{2}-b_{1} T t_{1}-\frac{b_{1} t_{1}^{2}}{2}\right)\right.$

$$
\left.+b\left(\frac{-5 b_{1} T^{3}}{6}-\frac{b_{1} T t_{1}^{2}}{2}-\frac{b_{1} t_{1}^{2}}{3}\right)-\frac{c b_{1} t_{1}^{3} T}{3}\right]
$$

Regarding the interest charged and interest earned, based on the values $\mathrm{M}, t_{w}, t_{0}$ we have the following three possible.

$$
\text { (i) } t_{0}>M \geq t_{w} \text {, (ii) } t_{0} \geq t_{w}>M \text { and (iii) } t_{w}>t_{0}>M
$$

\section{Case (i)}

In this case there are three sub - cases.

Sub-case (i): $\mathbf{M} \leq t_{1}$

Imbursement of the goods is matured and the vendor starts paying the capital opportunity cost for the items in store with rate $I_{p}$. Hence, the annual interest payable is

$$
\begin{aligned}
= & \frac{I_{p} C_{0}}{T a_{1}} \int_{M}^{t_{1}}\left(a+b t+c t^{2}\right)\left(e^{a_{1}\left(t_{1}-t\right)}-1\right) d t \\
= & -\frac{I_{p} C_{0}}{T a_{1}^{2}}\left[\left(a+b t_{1}+\frac{b}{a_{1}}+c t_{1}^{2}+\frac{2 c t_{1}}{a_{1}}+\frac{2 c}{a_{1}^{2}}\right)\right. \\
& \left.-\left(a e^{a_{1}\left(t_{1}-M\right)}+b M e^{a_{1}\left(t_{1}-t\right)}+b e^{a_{1}\left(t_{1}-t\right)}+c M^{2} e^{a_{1}\left(t_{1}-t\right)}+\frac{2 c M e^{a_{1}\left(t_{1}-t\right)}}{a_{1}}+\frac{2 c e^{a_{1}\left(t_{1}-t\right)}}{a_{1}^{2}}\right)\right]
\end{aligned}
$$

We assume that during the time the amount is not settled, the interest earned from the permissible period is $I_{e}$ per year.

Hence, $=\frac{I_{e} P}{T} \int_{0}^{M}\left(a+b t+c t^{2}\right) t d t$ 
Operations Research and Applications : An International Journal (ORAJ), Vol.2, No.4, November 2015

$$
=\frac{I_{e} P}{T} \int_{0}^{M}\left(a t+b t^{2}+c t^{3}\right) d t=\frac{I_{e} P}{T}\left(\frac{a M^{2}}{2}+\frac{b M^{3}}{3} \frac{c M^{4}}{4}\right)
$$

Sub-case (ii): $t_{w} \leq t_{1} \leq M$

In this case, there is no interest payable is charged by the retailer, but the interest earned is received by them. Therefore the interest payable is zero in this case and the annual interest earned is $I_{e}$ per year.

Hence,

$$
\begin{aligned}
& =\frac{I_{e} P}{T}\left[\int_{0}^{t_{1}}\left(a+b t+c t^{2}\right) t d t+\int_{0}^{t_{1}}\left(M-t_{1}\right)\left(a+b t+c t^{2}\right) d t\right] \\
& =\frac{I_{e} P}{T}\left[\left(a\left(M t_{1}-\frac{t_{1}^{2}}{2}\right)+b\left(\frac{M t_{1}^{2}}{2}-\frac{t_{1}^{3}}{6}\right)+\frac{c M t_{1}^{3}}{3}\right)\right]
\end{aligned}
$$

Sub-case (iii): $0<t_{1}<t_{w}$

If $t_{1}<t_{w}$, then the retailer must borrow the partial payment $(1-d) C_{0} Q$ at time zero and pay the suppliers and then pay off the loan from the sales revenue at time $\frac{(1-d) C_{0}}{P}\left(\frac{e^{a_{1} t_{1}}-1}{a_{1}}\right)$.From the time zero to $\frac{(1-d) C_{0}}{P}\left(\frac{e^{a_{1} t_{1}}-1}{a_{1}}\right)$ the annual interest charged

and payment is, the annual interest payable $=\frac{I_{e} C_{0}^{2}\left(a+b t+c t^{2}\right)(1-d)^{2}}{2 P T}\left(\frac{e^{a_{1} t_{1}}-1}{a_{1}}\right)^{2}$

And the annual interest earned $=\frac{I_{e} P}{2 T}\left[\left(a+b t+c t^{2}\right)\left(t_{1}-\frac{(1-d) C_{0}}{P}\left(\frac{e^{a_{1} t_{1}}-1}{a_{1}}\right)\right)\right]^{2}$

$+\frac{I_{e} P\left(M-t_{1}\right)}{T}\left[\left(a+b t+c t^{2}\right)\left(t_{1}-\frac{(1-d) C_{0}}{P}\left(\frac{e^{a_{1} t_{1}}-1}{a_{1}}\right)\right)\right]$

Thus, the total cost for the retailer can be expressed as, 
Operations Research and Applications : An International Journal (ORAJ), Vol.2, No.4, November 2015

$$
T C\left(t_{1}, T\right)= \begin{cases}T C_{1}\left(t_{1}, T_{1}\right) & \text { if } M \leq t_{1} \\ T C_{2}\left(t_{1}, T_{2}\right) & \text { if } t_{w} \leq t_{1} \leq M \\ T C_{3}\left(t_{1}, T_{3}\right) & \text { if } 0<t_{1}<t_{w}\end{cases}
$$

Now we calculate the values of $T C_{1}, T C_{2}, T C_{3}$.

Hence,

$$
\begin{aligned}
& T C_{1}\left(t_{1}, T\right)=\frac{A}{T_{1}}-\left(\frac{h+C_{0} a_{1}}{T_{1} a_{1}}\right)\left(a t_{1}+\frac{b t_{1}^{2}}{2}+\frac{c t_{1}^{3}}{3}-\frac{b t_{1}}{a_{1}}-\frac{c t_{1}^{2}}{a_{1}}+\frac{2 c t_{1}}{a_{1}^{2}}\right) \\
& -\frac{C_{1}}{T}\left[\left(\left(\frac{-a T^{2}}{2}\right)+\frac{a b_{1} T^{3}}{2}-\frac{a b_{1} T^{3}}{6}-\frac{b T^{3}}{6}+\frac{b b_{1} T^{4}}{6}\right)\right. \\
& \left.-\left(\frac{-a t_{1}^{2}}{2}+\frac{a b_{1} T t_{1}^{2}}{2}-\frac{a b_{1} t_{1}^{3}}{6}-\frac{b t_{1}^{3}}{6}+\frac{b t_{1}^{3} b_{1}}{6}\right)\right]+\frac{C_{2} b_{1}}{T_{1}}\left[a\left(\frac{T_{1}^{2}}{2}-T_{1} t_{1}-\frac{t_{1}^{2}}{2}\right)\right. \\
& \left.+b\left(\frac{-5 T_{1}^{3}}{6}-\frac{T_{1} t_{1}^{2}}{2}-\frac{t_{1}^{3}}{3}\right)-\frac{c t_{1}^{3} T_{1}}{3}\right]-\frac{I_{p} C_{0}}{T_{1} a_{1}^{2}}\left[\left(a+b t_{1}+\frac{b}{a_{1}}+c t_{1}^{2}+\frac{2 c t_{1}}{a_{1}}+\frac{2 c}{a_{1}^{2}}\right)\right. \\
& \left.-\left(a e^{a_{1}\left(t_{1}-M\right)}+b M e^{a_{1}\left(t_{1}-t\right)}+b e^{a_{1}\left(t_{1}-t\right)}+c M^{2} e^{a_{1}\left(t_{1}-t\right)}+\frac{2 c M e^{a_{1}\left(t_{1}-t\right)}}{a_{1}}+\frac{2 c e^{a_{1}\left(t_{1}-t\right)}}{a_{1}^{2}}\right)\right] \\
& -\frac{I_{e} P}{T_{1}}\left(\frac{a M^{2}}{2}+\frac{b M^{3}}{3} \frac{c M^{4}}{4}\right) \\
& T C_{2}\left(t_{1}, T_{2}\right)=\frac{A}{T_{2}}-\left(\frac{h+C_{0} a_{1}}{T_{2} a_{1}}\right)\left(a t_{1}+\frac{b t_{1}^{2}}{2}+\frac{c t_{1}^{3}}{3}-\frac{b t_{1}}{a_{1}}-\frac{c t_{1}^{2}}{a_{1}}+\frac{2 c t_{1}}{a_{1}^{2}}\right) \\
& -\frac{C_{1}}{T}\left[\left(\left(\frac{-a T^{2}}{2}\right)+\frac{a b_{1} T^{3}}{2}-\frac{a b_{1} T^{3}}{6}-\frac{b T^{3}}{6}+\frac{b b_{1} T^{4}}{6}\right)\right. \\
& \left.-\left(\frac{-a t_{1}^{2}}{2}+\frac{a b_{1} T t_{1}^{2}}{2}-\frac{a b_{1} t_{1}^{3}}{6}-\frac{b t_{1}^{3}}{6}+\frac{b t_{1}^{3} b_{1}}{6}\right)\right] \\
& +\frac{C_{2} b_{1}}{T_{2}}\left[a\left(\frac{T_{2}^{2}}{2}-T_{2} t_{1}-\frac{t_{1}^{2}}{2}\right)+b\left(\frac{-5 T_{2}^{3}}{6}-\frac{T_{2} t_{1}^{2}}{2}-\frac{t_{1}^{3}}{3}\right)-\frac{c t_{1}^{3} T_{2}}{3}\right] \\
& -\frac{I_{e} P}{T_{2}}\left[\left(a\left(M t_{1}-\frac{t_{1}^{2}}{2}\right)+b\left(\frac{M t_{1}^{2}}{2}-\frac{t_{1}^{3}}{6}\right)+\frac{c M t_{1}^{3}}{3}\right)\right]
\end{aligned}
$$


Operations Research and Applications : An International Journal (ORAJ), Vol.2, No.4, November 2015

$$
\begin{aligned}
T C_{3}\left(t_{1}, T_{3}\right)= & \frac{A}{T_{3}}-\left(\frac{h+C_{0} a_{1}}{T_{3} a_{1}}\right)\left(a t_{1}+\frac{b t_{1}^{2}}{2}+\frac{c t_{1}^{3}}{3}-\frac{b t_{1}}{a_{1}}-\frac{c t_{1}^{2}}{a_{1}}+\frac{2 c t_{1}}{a_{1}^{2}}\right) \\
& -\frac{C_{1}}{T}\left[\left(\left(\frac{-a T^{2}}{2}\right)+\frac{a b_{1} T^{3}}{2}-\frac{a b_{1} T^{3}}{6}-\frac{b T^{3}}{6}+\frac{b b_{1} T^{4}}{6}\right)\right. \\
& \left.-\left(\frac{-a t_{1}^{2}}{2}+\frac{a b_{1} T t_{1}^{2}}{2}-\frac{a b_{1} t_{1}^{3}}{6}-\frac{b t_{1}^{3}}{6}+\frac{b t_{1}^{3} b_{1}}{6}\right)\right]+\frac{C_{2} b_{1}}{T_{3}}\left[a\left(\frac{T_{3}^{2}}{2}-T_{3} t_{1}-\frac{t_{1}^{2}}{2}\right)\right. \\
& \left.+b\left(\frac{-5 T_{3}^{3}}{6}-\frac{T_{3} t_{1}^{2}}{2}-\frac{t_{1}^{3}}{3}\right)-\frac{c t_{1}^{3} T_{3}}{3}\right]+\frac{I_{e} C_{0}^{2}\left(a+b t+c t^{2}\right)(1-d)^{2}}{2 P T_{3}}\left(\frac{e^{a_{1} t_{1}}-1}{a_{1}}\right)^{2} \\
- & \frac{I_{e} P}{2 T_{3}}\left[\left(a+b t+c t^{2}\right)\left(t_{1}-\frac{(1-d) C_{0}}{P}\left(\frac{e^{a_{1} t_{1}}-1}{a_{1}}\right)\right)\right]^{2} \\
& -\frac{I_{e} P\left(M-t_{1}\right)\left[\left(a+b t+c t^{2}\right)\left(t_{1}-\frac{(1-d) C_{0}}{P}\left(\frac{e^{a_{1} t_{1}}-1}{a_{1}}\right)\right)\right]}{T_{3}}[12)
\end{aligned}
$$

\section{SOLUTION PROCESS}

To minimize the total cost, we determine the optimal storage cost and the optimal replenishment time.

Case 1: $t_{w} \leq t_{1} \leq M$

\section{Sub-case (i)}

Equation (10) is minimum when $\frac{\partial T C_{1}\left(t_{1}, T_{1}\right)}{\partial t_{1}}=0$ and $\frac{\partial T C_{1}\left(t_{1}, T_{1}\right)}{\partial T_{1}}=0$ which yields

$$
\begin{aligned}
\frac{\partial T C_{1}\left(t_{1}, T_{1}\right)}{\partial t_{1}} & \Rightarrow-\left(\frac{h+C_{0} a_{1}}{T_{1} a_{1}}\right)\left(a+b t_{1}+c t_{1}^{2}-\frac{b}{a_{1}}-\frac{2 c t_{1}}{a_{1}}+\frac{2 c}{a_{1}^{2}}\right) \\
& \left.-\frac{C_{1}}{T_{1}}\left[a\left(t_{1}+b_{1} T t_{1}-\frac{b_{1} t_{1}^{2}}{2}\right)-\frac{b t_{1}^{2}}{2}+\frac{b t_{1}^{2} b_{1}}{2}\right)\right] \\
& +\frac{C_{2} b_{1}}{T_{1}}\left[a\left(-T_{1}-t_{1}\right)+b\left(-T_{1} t_{1}-t_{1}^{2}\right)-c\left(t_{1}^{2} T_{1}\right)\right] \\
& -\frac{I_{p} C_{0}}{T_{1} a_{1}^{2}}\left[\left(b+2 c t_{1}+\frac{2 c}{a_{1}}\right)-\left(a a_{1} e^{a_{1}\left(t_{1}-M\right)}+b M a_{1} e^{a_{1}\left(t_{1}-M\right)}\right.\right. \\
& \left.\left.+b a_{1} e^{a_{1}\left(t_{1}-M\right)}+c M^{2} a_{1} e^{a_{1}\left(t_{1}-M\right)}+\frac{2 c M a_{1} e^{a_{1}\left(t_{1}-M\right)}}{a_{1}}+\frac{2 c a_{1} e^{a_{1}\left(t_{1}-M\right)}}{a_{1}^{2}}\right)\right]=0
\end{aligned}
$$


Operations Research and Applications : An International Journal (ORAJ), Vol.2, No.4, November 2015

$$
\begin{aligned}
& \frac{\partial T C_{1}\left(t_{1}, T_{1}\right)}{\partial T_{1}} \Rightarrow-\frac{A}{T_{1}^{2}}+\left(\frac{h+C_{0} a_{1}}{T_{1}^{2} a_{1}}\right)\left(a t_{1}+\frac{b t_{1}^{2}}{2}+\frac{c t_{1}^{3}}{3}-\frac{b t_{1}}{a_{1}}-\frac{c t_{1}^{2}}{a_{1}}+\frac{2 c t_{1}}{a_{1}^{2}}\right) \\
& \quad+\frac{C_{1} a T^{2}}{2}+\frac{2 C_{1} a T^{3} b_{1}}{6}-\frac{C_{1} b T^{3}}{6}+\frac{C_{1} a t_{1}^{2}}{2}+\frac{C_{1} a b_{1} t_{1}^{3}}{6} \\
& +\frac{C_{1} b t_{1}^{3}}{6}-\frac{C_{1} b t_{1}^{3} b_{1}}{6}-\frac{3 a C_{1} T^{2} b_{1}}{2}+\frac{C_{1} a b_{1} T^{2}}{2}-\frac{C_{1} b t_{1}^{3} b_{1}}{6} \\
& -\frac{C_{2} b_{1}}{T_{1}^{2}}\left[a\left(\frac{T_{1}^{2}}{2}-T_{1} t_{1}-\frac{t_{1}^{2}}{2}\right)+b\left(\frac{-5 T_{1}^{3}}{6}-\frac{T_{1} t_{1}^{2}}{2}-\frac{t_{1}^{3}}{3}\right)-\frac{c t_{1}^{3} T_{1}}{3}\right] \\
& +\frac{C_{2} b_{1}}{T_{1}}\left[a\left(T_{1}-t_{1}\right)+b\left(\frac{-5 T_{1}^{2}}{2}-\frac{t_{1}^{2}}{2}\right)-\frac{c t_{1}^{3}}{3}\right] \\
& +\frac{I_{p} C_{0}}{T_{1}^{2} a_{1}^{2}}\left[\left(a+b t_{1}+\frac{b}{a_{1}}+c t_{1}^{2}+\frac{2 c t_{1}}{a_{1}}+\frac{2 c}{a_{1}^{2}}\right)\right. \\
& -\left(a e^{a_{1}\left(t_{1}-M\right)}+b M e^{a_{1}\left(t_{1}-M\right)}+b e^{a_{1}\left(t_{1}-M\right)}\right. \\
& \left.\left.+\frac{2 c M e^{a_{1}\left(t_{1}-M\right)}}{a_{1}}+\frac{2 c e^{a_{1}\left(t_{1}-M\right)}}{a_{1}^{2}}\right)\right] \\
& +\frac{I_{e} P}{T_{1}^{2}}\left[\frac{a M^{2}}{2}+\frac{b M^{3}}{3}\right]=0
\end{aligned}
$$

From the above equation, now we calculate the value of $T_{1}^{2}$,

$$
\begin{aligned}
& T_{1}^{2}=\left[2 \left(A-\left(\frac{h+C_{0} a_{1}}{a_{1}}\right)\left(a t_{1}+\frac{b t_{1}^{2}}{2}+\frac{c t_{1}^{3}}{3}-\frac{b t_{1}}{a_{1}}-\frac{c t_{1}^{2}}{a_{1}}+\frac{2 c t_{1}}{a_{1}^{2}}\right)\right.\right. \\
& +\frac{C_{1} a t_{1}^{2}}{2}+\frac{C_{1} a b_{1} t_{1}^{3}}{6}+\frac{C_{1} b t_{1}^{3}}{6}-\frac{C_{1} b t_{1}^{3} b_{1}}{6} \\
& +\frac{C_{2} b_{1} a t_{1}^{2}}{2}+\frac{C_{2} b b_{1} t_{1}^{3}}{3}-\frac{I_{o} C_{0}}{a_{1}^{2}}\left[\left[\left(a+b t+c t_{1}^{2}+\frac{b}{a_{1}}+\frac{2 c t_{1}}{a_{1}}+\frac{2 c}{a_{1}^{3}}\right)\right.\right. \\
& \left.-\left(a e^{a_{1}\left(t_{1}-M\right)}+b M e^{a_{1}\left(t_{1}-M\right)}+b e^{a_{1}\left(t_{1}-M\right)}+c M^{2} e^{a_{1}\left(t_{1}-M\right)}+\frac{2 c e^{a_{1}\left(t_{1}-M\right)}}{a_{1}}+\frac{2 c e^{a_{1}\left(t_{1}-M\right)}}{a_{1}^{2}}\right)\right] \\
& \left.-I_{e} P\left(\frac{a M^{2}}{2}+\frac{b M^{3}}{3}\right)\right] \div\left[C_{1} a-3 C_{1} a b_{1}-C_{1} b+C_{2} b_{1} a\right]
\end{aligned}
$$


Operations Research and Applications : An International Journal (ORAJ), Vol.2, No.4, November 2015

From equation (15) we have to calculate the value of $T_{1}$

$$
\begin{aligned}
& T_{1}=S q r t\left(\left[2 \left(A-\left(\frac{h+C_{0} a_{1}}{a_{1}}\right)\left(a t_{1}+\frac{b t_{1}^{2}}{2}+\frac{c t_{1}^{3}}{3}-\frac{b t_{1}}{a_{1}}-\frac{c t_{1}^{2}}{a_{1}}+\frac{2 c t_{1}}{a_{1}^{2}}\right)\right.\right.\right. \\
& +\frac{C_{1} a t_{1}^{2}}{2}+\frac{C_{1} a b_{1} t_{1}^{3}}{6}+\frac{C_{1} b t_{1}^{3}}{6}-\frac{C_{1} b t_{1}^{3} b_{1}}{6}+\frac{C_{2} b_{1} a t_{1}^{2}}{2}+\frac{C_{2} b b_{1} t_{1}^{3}}{3} \\
& -\frac{I_{o} C_{0}}{a_{1}^{2}}\left[\left[\left(a+b t+c t_{1}^{2}+\frac{b}{a_{1}}+\frac{2 c t_{1}}{a_{1}}+\frac{2 c}{a_{1}^{3}}\right)\right.\right. \\
& \left.-\left(a e^{a_{1}\left(t_{1}-M\right)}+b M e^{a_{1}\left(t_{1}-M\right)}+b e^{a_{1}\left(t_{1}-M\right)}+c M^{2} e^{a_{1}\left(t_{1}-M\right)}+\frac{2 c e^{a_{1}\left(t_{1}-M\right)}}{a_{1}}+\frac{2 c e^{a_{1}\left(t_{1}-M\right)}}{a_{1}^{2}}\right)\right] \\
& \left.-I_{e} P\left(\frac{a M^{2}}{2}+\frac{b M^{3}}{3}\right)\right] \div\left[C_{1} a-3 C_{1} a b_{1}-C_{1} b+C_{2} b_{1} a\right]
\end{aligned}
$$

\section{Sub-case (ii)}

Equation (11) is minimum when $\frac{\partial T C_{2}\left(t_{1}, T_{2}\right)}{\partial t_{1}}=0$ and $\frac{\partial T C_{2}\left(t_{1}, T_{2}\right)}{\partial T_{2}}=0$ which yields

$$
\begin{aligned}
& \frac{\partial T C_{2}\left(t_{1}, T_{2}\right)}{\partial t_{1}} \Rightarrow-\left(\frac{h+C_{0} a_{1}}{T_{2} a_{1}}\right)\left(a+b t_{1}+c t_{1}^{2}-\frac{b}{a_{1}}-\frac{2 c t_{1}}{a_{1}}+\frac{2 c}{a_{1}^{2}}\right) \\
& \left.-\frac{C_{1}}{T_{1}}\left[a\left(t_{1}+b_{1} T t_{1}-\frac{b_{1} t_{1}^{2}}{2}\right)-\frac{b t_{1}^{2}}{2}+\frac{b t_{1}^{2} b_{1}}{2}\right)\right] \quad+\frac{C_{2} b_{1}}{T_{2}}\left[a\left(-T_{2}-t_{1}\right)+b\left(-T_{2} t_{1}-t_{1}^{2}\right)\right.
\end{aligned}
$$

$$
\begin{gathered}
\left.-c\left(t_{1}^{2} T_{2}\right)\right] \\
-\frac{I_{e} P}{T_{2}}\left[a\left(M-t_{1}\right)+b\left(M t_{1}-\frac{t_{1}^{2}}{2}\right)+c M t_{1}^{2}\right]=0 \\
\frac{\partial T C_{2}\left(t_{1}, T_{2}\right)}{\partial T_{2}} \Rightarrow-\frac{A}{T_{2}^{2}}+\left(\frac{h+C_{0} a_{1}}{T_{2}^{2}}\right)\left(a t_{1}+\frac{b t_{1}^{2}}{2}+\frac{c t_{1}^{3}}{3}-\frac{b t_{1}}{a_{1}}-\frac{c t_{1}^{2}}{a_{1}}+\frac{2 c t_{1}}{a_{1}^{2}}\right) \\
+\frac{C_{1} a T^{2}}{2}+\frac{2 C_{1} a T^{3} b_{1}}{6}-\frac{C_{1} b T^{3}}{6}+\frac{C_{1} a t_{1}^{2}}{2}+\frac{C_{1} a b_{1} t_{1}^{3}}{6} \\
+\frac{C_{1} b t_{1}^{3}}{6}-\frac{C_{1} b t_{1}^{3} b_{1}}{6}-\frac{3 a C_{1} T^{2} b_{1}}{2}+\frac{C_{1} a b_{1} T^{2}}{2}-\frac{C_{1} b t_{1}^{3} b_{1}}{6} \\
+\frac{C_{2}}{T_{2}^{2}}\left[a\left(\frac{b_{1} T_{2}^{2}}{2}-b_{1} T_{2} t_{1}-\frac{b_{1} t_{1}^{2}}{2}\right)+b\left(\frac{-5 b_{1} T_{2}^{3}}{6}-\frac{b_{1} T_{2} t_{1}^{2}}{2}-\frac{b_{1} t_{1}^{3}}{3}\right)+\frac{c b_{1} t_{1}^{3} T_{2}}{3}\right]
\end{gathered}
$$


Operations Research and Applications : An International Journal (ORAJ), Vol.2, No.4, November 2015

$$
\begin{aligned}
& +\frac{C_{2}}{T_{2}}\left[a\left(b_{1} T_{2}-b_{1} t_{1}\right)+b\left(\frac{-5 b_{1} T_{2}^{2}}{2}-\frac{b_{1} t_{1}^{2}}{2}\right)-\frac{c b_{1} t_{1}^{3}}{3}\right] \\
& +\frac{I_{e} P}{T_{2}^{2}}\left[a\left(M t_{1}-\frac{t_{1}^{2}}{2}\right)+b\left(\frac{M t_{1}^{2}}{2}-\frac{t_{1}^{3}}{6}\right)+\frac{c M t_{1}^{3}}{3}\right]=0
\end{aligned}
$$

From the above equation we calculate the value of $T_{2}^{2}$

$$
\begin{aligned}
T_{2}^{2}= & {\left[2 \left(A-\left(\frac{h+C_{0} a_{1}}{a_{1}}\right)\left(a t_{1}+\frac{b t_{1}^{2}}{2}+\frac{c t_{1}^{3}}{3}-\frac{b t_{1}}{a_{1}}-\frac{c t_{1}^{2}}{a_{1}}+\frac{2 c t_{1}}{a_{1}^{2}}\right)+\frac{C_{1} a t_{1}^{2}}{2}+\frac{C_{1} a b_{1} t_{1}^{3}}{6}+\frac{C_{1} b t_{1}^{3} b_{1}}{6}\right.\right.} \\
& +\frac{C_{2} a b_{1} t_{1}^{2}}{2}+I_{e} P\left[a\left(M t_{1}-\frac{t_{1}^{2}}{2}\right)+b\left(\frac{M t_{1}^{2}}{2}-\frac{t_{1}^{3}}{6}\right)+\frac{c M t_{1}^{3}}{3}\right] \div\left(-3 C_{1} a-C_{2} a b_{1} t_{1}\right)
\end{aligned}
$$

From equation (19) we get value of $T_{2}$,

$$
\begin{aligned}
& T_{2}=\operatorname{Sqrt}\left[2 \left(A-\left(\frac{h+C_{0} a_{1}}{a_{1}}\right)\left(a t_{1}+\frac{b t_{1}^{2}}{2}+\frac{c t_{1}^{3}}{3}-\frac{b t_{1}}{a_{1}}-\frac{c t_{1}^{2}}{a_{1}}+\frac{2 c t_{1}}{a_{1}^{2}}\right)\right.\right. \\
& +\frac{C_{1} a t_{1}^{2}}{2}+\frac{C_{1} a b_{1} t_{1}^{3}}{6}+\frac{C_{1} b t_{1}^{3} b_{1}}{6} \\
& \quad+\frac{C_{2} a b_{1} t_{1}^{2}}{2}+I_{e} P\left[a\left(M t_{1}-\frac{t_{1}^{2}}{2}\right)+b\left(\frac{M t_{1}^{2}}{2}-\frac{t_{1}^{3}}{6}\right)+\frac{c M t_{1}^{3}}{3}\right] \div\left(-3 C_{1} a-C_{2} a b_{1} t_{1}\right)
\end{aligned}
$$

Sub-case (iii)

Equation (12) is minimum when $\frac{\partial T C_{3}\left(t_{1}, T_{3}\right)}{\partial t_{1}}=0$ and $\frac{\partial T C_{3}\left(t_{1}, T_{3}\right)}{\partial T_{3}}=0$ which yield

$$
\begin{aligned}
& \frac{\partial T C_{3}\left(t_{1}, T_{3}\right)}{\partial t_{1}} \Rightarrow-\left(\frac{h+C_{0} a_{1}}{T_{3} a_{1}}\right)\left(a+b t_{1}+c t_{1}^{2}-\frac{b}{a_{1}}-\frac{2 c t_{1}}{a_{1}}+\frac{2 c}{a_{1}^{2}}\right) \\
& \left.-\frac{C_{1}}{T_{1}}\left[a\left(t_{1}+b_{1} T t_{1}-\frac{b_{1} t_{1}^{2}}{2}\right)-\frac{b t_{1}^{2}}{2}+\frac{b t_{1}^{2} b_{1}}{2}\right)\right]+\frac{C_{2} b_{1}}{T_{3}}\left[a\left(-T_{3}-t_{1}\right)+b\left(-T_{3} t_{1}-t_{1}^{2}\right)-c\left(t_{1}^{2} T_{3}\right)\right] \\
& +\frac{I_{e} P}{2 T_{3}}\left(a+b t+c t^{2}\right) \\
& \quad\left[2 t_{1}-2\left(\frac{(1-d) C_{0}}{P a_{1}}\left(\frac{t_{1} e^{a_{t_{1}}}}{a_{1}}\right)+2\left(\frac{(1-d) C_{0}}{P a_{1}}\left(\frac{e^{a_{1} t_{1}}}{a_{1}^{2}}\right)\right)+\frac{2(1-d) C_{0}}{P a_{1}}\right]=0\right. \\
& \frac{\partial T C_{2}\left(t_{1}, T_{3}\right)}{\partial T_{3}} \Rightarrow-\frac{A}{T_{3}^{2}}+\left(\frac{h+C_{0} a_{1}}{T_{3}^{2}}\right)\left(a t_{1}+\frac{b t_{1}^{2}}{2}+\frac{c t_{1}^{3}}{3}-\frac{b t_{1}}{a_{1}}-\frac{c t_{1}^{2}}{a_{1}}+\frac{2 c t_{1}}{a_{1}^{2}}\right)
\end{aligned}
$$


Operations Research and Applications : An International Journal (ORAJ), Vol.2, No.4, November 2015

$$
\begin{aligned}
& +\left(\frac{C_{1}}{T_{3}^{2}}\right)\left[a\left(\frac{T_{3}^{2}}{2}+\frac{a b_{1} T_{3}^{2}}{2}-\frac{a b_{1} T_{3}^{3}}{6}\right)-\frac{b T_{3}^{3}}{6}\right) \\
& \left.-\left(-\frac{a t_{1}^{2}}{2}+\frac{a b_{1} t_{1}^{2} T_{3}}{2}-\frac{a b_{1} t_{1}^{3}}{6}-\frac{b t_{1}^{3} b_{1}}{6}\right)\right]-\frac{C_{1}}{T_{3}}\left[a\left(-T_{3}+\frac{3 a b_{1} T^{2}}{2}-\frac{3 a b_{1} T^{2}}{6}-\frac{3 b T^{2}}{6}-\frac{a b_{1} t_{1}^{2}}{2}\right)\right. \\
& +\frac{C_{2}}{T_{3}^{2}}\left[a\left(\frac{b_{1} T_{3}^{2}}{2}-b_{1} T_{3} t_{1}-\frac{b_{1} t_{1}^{2}}{2}\right)+b\left(\frac{-5 b_{1} T_{3}^{3}}{6}-\frac{b_{1} T_{3} t_{1}^{2}}{2}-\frac{b_{1} t_{1}^{3}}{3}\right)+\frac{c b_{1} t_{1}^{3} T_{3}}{3}\right]+\frac{C_{2}}{T_{3}}\left[a\left(b_{1} T_{3}-b_{1} t_{1}\right)\right. \\
& \left.+b\left(\frac{-5 b_{1} T_{3}^{2}}{2}-\frac{b_{1} t_{1}^{2}}{2}\right)-\frac{c b_{1} t_{1}^{3}}{3}\right]-\frac{I_{p} C_{0}^{2}\left(a+b t+c t^{2}\right)(1-d)^{2}}{2 P T_{3}^{2}}\left(\frac{e^{a_{1} t_{1}}-1}{a_{1}}\right)^{2}+\frac{I_{e} P}{2 T^{2}}\left[\left(a+b t+c t^{2}\right)\right. \\
& \left(t_{1}-\frac{(1-d) C_{0}}{P}\left(\frac{e^{a_{1} t_{1}}-1}{a_{1}}\right)\right)^{2}+\frac{I_{e} P\left(M-t_{1}\right)}{T^{2}}\left[\left(a+b t+c t^{2}\right)\right. \\
& \left(t_{1}-\frac{(1-d) C_{0}}{P}\left(\frac{e^{a_{1} t_{1}}-1}{a_{1}}\right)\right)=0
\end{aligned}
$$

From the above equation we calculate the value of $T_{3}^{2}$,

$$
\begin{aligned}
T_{3}^{2} & =\left[2 \left(-A+\left(\frac{h+C_{0} a_{1}}{a_{1}}\right)\left(a t_{1}+\frac{b t_{1}^{2}}{2}+\frac{c t_{1}^{3}}{3}-\frac{b t_{1}}{a_{1}}-\frac{c t_{1}^{2}}{a_{1}}+\frac{2 c t_{1}}{a_{1}^{2}}\right)\right.\right. \\
+ & \frac{C_{1} a t_{1}^{2}}{2}+\frac{C_{1} a b_{1} t_{1}^{3}}{6}+\frac{C_{1} b t_{1}^{3}}{6}-\frac{C_{1} b b_{1} t_{1}^{3}}{6}+\frac{C_{1} a b_{1} t_{1}^{2}}{2}+\frac{C_{2} a b_{1} t_{1}^{2}}{2}+\frac{C_{2} b b_{1} t_{1}^{3}}{3} \\
& -\frac{I_{p} C_{0}^{2}\left(a+b t+c t^{2}\right)}{2 P}(1-d)^{2}\left(\frac{e^{a_{1} t_{1}}-1}{a_{1}}\right)^{2}-\frac{I_{e} P}{2}\left[\left(a+b t+c t^{2}\right)\left(t_{1}-\frac{(1-d) C_{0}}{P}\left(\frac{e^{a_{1} t_{1}}-1}{a_{1}}\right)\right)\right]^{2} \\
& \left.-I_{e} P\left(M-t_{1}\right)\left(a+b t+c t^{2}\right)\left(t_{1}-\frac{(1-d) C_{0}}{P}\left(\frac{e^{a_{1} t_{1}}-1}{a_{1}}\right)\right)\right] \\
& \div\left(-C_{1} a-C_{2} a b_{1}+5 C_{2} b b_{1}\right)
\end{aligned}
$$

From the equation (23) we calculate $T_{3}$,

$$
\begin{aligned}
T_{3}=\operatorname{sqrt}\left[2 \left(-A+\left(\frac{h+C_{0} a_{1}}{a_{1}}\right)\left(a t_{1}+\frac{b t_{1}^{2}}{2}+\frac{c t_{1}^{3}}{3}-\frac{b t_{1}}{a_{1}}-\frac{c t_{1}^{2}}{a_{1}}+\frac{2 c t_{1}}{a_{1}^{2}}\right)\right.\right. \\
+\frac{C_{1} a t_{1}^{2}}{2}+\frac{C_{1} a b_{1} t_{1}^{3}}{6}+\frac{C_{1} b t_{1}^{3}}{6}-\frac{C_{1} b b_{1} t_{1}^{3}}{6}+\frac{C_{1} a b_{1} t_{1}^{2}}{2}+\frac{C_{2} a b_{1} t_{1}^{2}}{2}+\frac{C_{2} b b_{1} t_{1}^{3}}{3}
\end{aligned}
$$


Operations Research and Applications : An International Journal (ORAJ), Vol.2, No.4, November 2015

$$
\begin{aligned}
& -\frac{I_{p} C_{0}^{2}\left(a+b t+c t^{2}\right)}{2 P}(1-d)^{2}\left(\frac{e^{a_{1} t_{1}}-1}{a_{1}}\right)^{2}-\frac{I_{e} P}{2}\left[\left(a+b t+c t^{2}\right)\left(t_{1}-\frac{(1-d) C_{0}}{P}\left(\frac{e^{a_{1} t_{1}}-1}{a_{1}}\right)\right]^{2}\right. \\
& \left.-I_{e} P\left(M-t_{1}\right)\left(a+b t+c t^{2}\right)\left(t_{1}-\frac{(1-d) C_{0}}{P}\left(\frac{e^{a_{1} t_{1}}-1}{a_{1}}\right)\right)\right] \div\left(-C_{1} a-C_{2} a b_{1}+5 C_{2} b b_{1}\right)
\end{aligned}
$$

\section{STATISTICAL EXAMPLES}

In order to demonstrate the solution method, let us consider an inventory system with the following data: Let $A=\$ 500$ per order, $h=\$ 15$ per unit per order, $M=2 / 12, C 1=\$ 20$ per unit, $\mathrm{C} 2=\$ 30$ per unit, $\mathrm{P}=\$ 85$ per unit, $I_{p}=0.15$ per dollar per year, $I_{e}=0.12$ per dollar per year, $a_{1}=0.08, b_{1}=0.50, \mathrm{~d}=0.8, \mathrm{w}=200, \mathrm{C}_{0}=25, \mathrm{a}=55, \mathrm{~b}=65, \mathrm{c}=75$, and $\mathrm{t}=2$ years

\begin{tabular}{|c|c|c|c|c|c|c|c|c|}
\hline $\mathrm{a}$ & $\mathrm{b}$ & $\mathrm{c}$ & $\mathrm{T}_{1}$ & $\mathrm{~T}_{2}$ & $\mathrm{~T}_{3}$ & $\mathrm{TC}_{1}$ & $\mathrm{TC}_{2}$ & $\mathrm{TC}_{3}$ \\
\hline 5 & 10 & 20 & 1.3576 & 1.4882 & 3.5176 & 77.5000 & 80.022 & 183.680 \\
\hline \multicolumn{3}{|l|}{$\mathrm{t}_{\mathrm{w}}$} & 16667 & 1.8524 & 2.8286 & & & \\
\hline 15 & 20 & 25 & & 1.3457 & 3.4020 & 201.2000 & 108.37 & 354.22 \\
\hline \multicolumn{3}{|l|}{$\mathrm{t}_{\mathrm{w}}$} & 1.0195 & 1.2407 & 2.1294 & & & \\
\hline 15 & 25 & 35 & 0.3054 & 2.7949 & 3.3652 & 272 & 532.85 & 518.70 \\
\hline \multicolumn{3}{|l|}{$\mathrm{t}_{\mathrm{w}}$} & 0.0668 & 0.0574 & 0.1936 & & & \\
\hline 55 & 65 & 75 & 1.8637 & 1.9394 & 4.4971 & 100.23 & 125.56 & 700.99 \\
\hline \multicolumn{3}{|l|}{$t_{w}$} & 0.3959 & 0.4078 & 0.9591 & & & \\
\hline
\end{tabular}

Table 1 Illustration of the solution procedure for the numerical example 
Operations Research and Applications : An International Journal (ORAJ), Vol.2, No.4, November 2015

\section{COMPASSION STUDY}

Table 2 Computational outcome with respect to different values of $h, A, M$

\begin{tabular}{|c|c|c|c|c|c|c|c|}
\hline $\begin{array}{l}\text { Changing } \\
\text { parameters }\end{array}$ & $\begin{array}{l}\text { Change in } \\
\text { parameters }\end{array}$ & $T_{1}$ & $T_{2}$ & $T_{3}$ & $T C_{1}$ & $T C_{2}$ & $T C_{3}$ \\
\hline \multirow{3}{*}{ h } & 20 & 2.6601 & 2.566 & 0.8790 & 258.55 & 722.37 & 711.13 \\
\hline & 25 & 3.3463 & 2.7724 & 0.9790 & 100.270 & 80.486 & 87.201 \\
\hline & 30 & 3.6205 & 2.9700 & 0.7050 & 102.93 & 87.884 & 152.19 \\
\hline \multirow{3}{*}{ A } & 300 & 2.6601 & 2.566 & 0.8790 & 258.55 & 722.37 & 711.13 \\
\hline & 350 & 3.3453 & 2.7734 & 0.9798 & 100.30 & 80.490 & 87.221 \\
\hline & 400 & 3.6225 & 2.9715 & 0.7055 & 102.97 & 87.897 & 152.25 \\
\hline \multirow{3}{*}{ M } & $1 / 12$ & 2.6601 & 2.566 & 0.8790 & 258.55 & 722.37 & 711.13 \\
\hline & $2 / 12$ & 3.3443 & 2.7730 & 0.9791 & 100.10 & 80.483 & 87.211 \\
\hline & $3 / 12$ & 3.6200 & 2.9710 & 0.7047 & 102.80 & 87.880 & 152.20 \\
\hline
\end{tabular}


Operations Research and Applications : An International Journal (ORAJ), Vol.2, No.4, November 2015

Table 3 Computational results with respect to different values of $b_{1}, a_{1}, I_{e}, I_{p}$

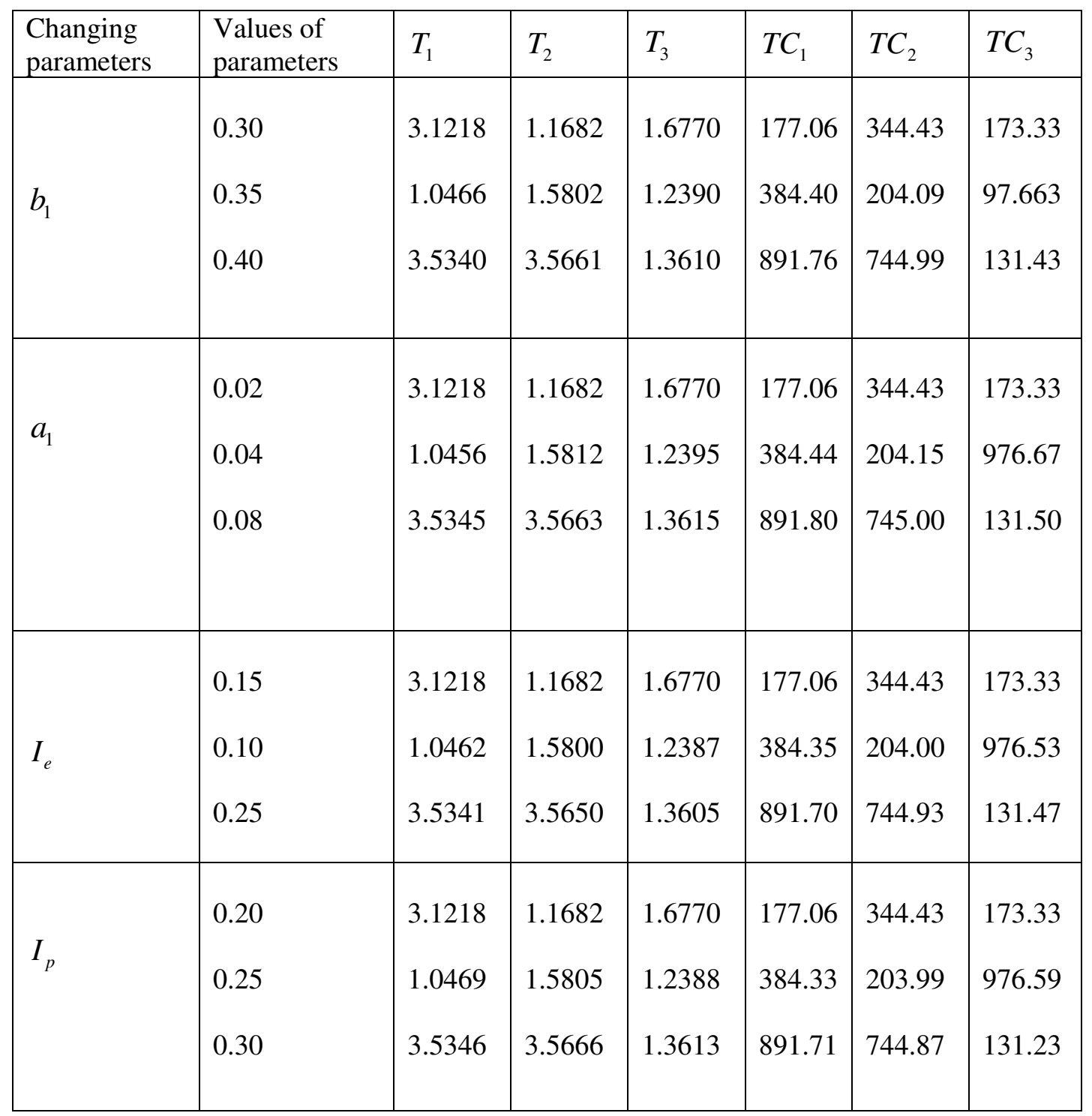

\section{TERMINATION}

In this paper, we have examined the partial trade credit financing in a supply chain by EOQ-based model for decomposing items together with shortages. We also assumed that the demand is quadratic. A mathematical is developed for the optimal solution with the shortage point, ordering quantity in order to minimize the total cost. A Statistical example is presented to demonstrate the solution process and the compassion study for various values of the parameters also offered. The proposed model can be further extended in it several ways. For example, it may be extended to multiple items with partial backlogging and also developing the model to changing the demand as cubic. 
Operations Research and Applications : An International Journal (ORAJ), Vol.2, No.4, November 2015

\section{ACKNOWLEDGEMENTS}

This research was fully supported by National Board of Higher Mathematics, Government of India under the scheme of NBHM research project with $2 / 48(9) / 2013 / \mathrm{NBHM}(\mathrm{R} . \mathrm{P}) / \mathrm{R} \& \mathrm{D}$ II /Dated 16.01.2014.

\section{REFERENCES}

[1] Abubakar M., Babangida S. (2012). Inventory ordering policies of delayed deteriorating items under permissible delay in payments, International Journal of Production Economics, 136:75- 83.

[2] Aggarwal, S. P. (1978). A note on an order-level inventory model for a system with constant rate of deterioration, Opsearch, 15:84-187.

[3] Amutha, R., Chandrasekaran, E. (2013). An EOQ model for deteriorating items with quadratic demand and time dependent holding cost, International Journal of Emerging Science and Engineering (IJESE) 1:5-6.

[4] Annadurai, K., Uthayakumar, R. (2012). Analysis of partial trade credit financing in a supply chain by EOQ-based model for decaying items with shortages, International Journal of Emerging Science and Engineering (IJESE) 61:1139-1159.

[5] Begum, R., Sahu, S. K. and Rsahoo, R. (2010). An EOQ model for deteriorating Items with Weibull Distribution Deterioration, Unit production cost with Quadratic demand and Shortages, Applied Mathematical Sciences, 4:271-288.

[6] Chaitanya Kumar Tripathy and Umakanta Mishra, (2010). Ordering Policy for Weibull Deteriorating Items for Quadratic Demand with Permissible Delay in Payments, Applied Mathematical Sciences, 4:2181 - 2191.

[7] Deb, M., Chaudhuri, K. (1987). A note on the heuristic for replenishment of trended inventories considering shortages, Journal of operations research Society, 38:459-463.

[8] Ghrae, P. M. and Schrader, G. F. (1963). An Inventory model for exponentially deteriorating items, Journal of Industrial Engineering, 14:238-243.

[9] Goyal, S. K., Giri, B.C. (2001) .Recent trends in modeling of deteriorating inventory, E. Journal of Operations research 134:1-16.

[10] Hardik N. Soni (2013). Optimal replenishment policies for deteriorating items with stock sensitive demand under two-level trade credit and limited capacity, Applied Mathematical Modelling,37:5887-5895.

[11] Hwang, H., Shinn, S. W. (1997). Retailer's pricing and lot-sizing policy for exponentially deteriorating products under the condition of permissible delay in payments. Computers and Operations Research, 24:539-547.

[12] Mitra, A., Fox, J.F. and Jessejr, R.R. (1980). A note on determining order quantities with a linear trend in demand. Journal of Operations Research Society, 31:15-20.

[13] Mohan, R.,Venkateswarlu, and R. January (2013). Inventory Management Model with Quadratic Demand, Variable Holding Cost with Salvage value, Journal of Management Sciences, 3:18-22.

[14] Liao, J. J. (2008). An EOQ model with non instantaneous receipt and exponential deteriorating item under two-level trade credit. International Journal of Production Economics 113:852-861.

[15] Sarkar, T. Ghosh, S. K. and Chaudhuri, K.S. ( 2013). An optimal inventory replenishment policy for a deteriorating item with time-quadratic demand and time-dependent partial backlogging with shortages in all cycles, Applied Mathematics and Computation,2 18: 9147-9155.

[16] Shital S. Patel1., Raman Patel. (2013). an inventory model for weibull deteriorating items with linear demand, shortages under permissible delay in payments and inflation, International Journal of Mathematics and Statistics Invention, 6:508-529

[17] Soni, H., Shah, N. H. (2008). Optimal ordering policy for stock dependent demand under progressive payment scheme. European Journal of Operations Research 184:91-100. 
Operations Research and Applications : An International Journal (ORAJ), Vol.2, No.4, November 2015

[18] Vandana., B. K. Sharma (2015). An EPQ inventory model for non-instantaneous deteriorating item sunder trade credit policy, International Journal of Mathematical Sciences and Industrial Applications (IJMSEA), 9:179-188.

[19] Vandana, B. K. Sharma (2015). An inventory model for Non- Instantaneous deteriorating items with quadratic demand rate and shortages under trade credit policy, Journal of Applied Analysis and Computation, in press. 